\title{
La Teoría de los Fundamentos Morales y la orientación política detrás del apoyo o rechazo al movimiento estudiantil chileno del 2011*
}

\section{The Moral Foundations Theory and the Political Orientation behind the Support or Rejection to the Chilean Student Movement of 2011}

Recepción: 26 Febrero 2017 | Aceptación: 05 Junio 2019

\author{
Dante Junior Solano Silva \\ University of Leeds, Reino Unido \\ ORCID: http://orcid.org/0000-0003-0008-2037 \\ IGNACIO CARRERE \\ Universidad de La Frontera, Chile \\ ORCID: http://orcid.org/0000-0002-1090-7015
}

a Autor de correspondencia. Correo electrónico: pt18djss@leeds.ac.uk

Para citar este artículo: Solano, D. J., \& Carrere, I. (2019). La Teoría de los Fundamentos Morales y la orientación política detrás del apoyo o rechazo al movimiento estudiantil chileno del 2011. Universitas Psychologica, 18(3), 1-12. https://doi.org/10.11144/ Javeriana.upsy18-3.tfmo

\section{RESUMEN}

La presente investigación estudia los fundamentos morales relacionados con el apoyo o rechazo al Movimiento Estudiantil Chileno (MECh) durante las manifestaciones del $2011(\mathrm{~N}=170)$. De acuerdo a los análisis factoriales -exploratorios y confirmatorios-, los fundamentos de daño/ cuidado, justicia/engaño, autoridad/subversión y santidad/degradación se comportaron consistentes con la teoría; pero el fundamento de lealtad/ traición, se dividió en dos valores: Patriotismo y Lealtad. Los fundamentos de Daño/cuidado y Justicia/engaño se asociaron a posiciones de la izquierda política y al apoyo al MECh; mientras que el patriotismo, la autoridad/subversión y la santidad/degradación se asociaron a la derecha y el rechazo a este. Un modelo de ecuaciones estructurales evidenció que el daño/cuidado se asociaba directamente al apoyo al movimiento; mientras que la justicia/engaño y la autoridad/subversión estaban mediadas por la orientación política.

Palabras clave

teoría de los fundamentos morales; movimiento estudiantil chileno; orientación política; actitudes hacia las políticas y movimientos sociales; sociedad chilena.

\footnotetext{
ABSTRACT

The research studies the moral foundations related to the support or rejection of the Chilean Student Movement MECh, during the manifestations of $2011(\mathrm{~N}=170)$. According to the factorial analysis exploratory and confirmatory-, the foundations of Justice/cheating, harm/ care, authority/subversion, and sanctity/degradation behaved consistently with the theory; but loyalty/betrayal was divided in two values: patriotism and loyalty. justice/cheating and harm/care were associated with the political left and the support to the movement. Patriotism, authority/ subversion, and sanctity/degradation were associated with the right and the rejection to the movement. A structural equation model reveals that harm/care was directly related to the support to movement; while justice/ cheating and authority/subversion was mediated by political orientation.
} 
Keywords

moral foundations theory; Chilean student movement; political orientation; attitudes towards policies and social movements; Chilean society.

Durante el año 2011, se desarrollaron importantes movilizaciones sociales en Chile en protesta al sistema educativo que se mantenía desde la década de los ochenta, época de la dictadura militar de Augusto Pinochet (Urra, 2012). Este movimiento de protesta, liderado por estudiantes universitarios y conocido como el Movimiento Estudiantil Chileno MECh, convocó a diversas organizaciones civiles chilenas (Urra, 2012), y tuvo un importante respaldo de la ciudadanía —un $89 \%$ de chilenos llegó a mostrarse a favor de la movilización (Centro de Estudios de la Realidad Contemporánea, 2011). Entender los mecanismos psicológicos subyacentes a las actitudes de la ciudadanía sobre temas sociopolíticos es uno de los objetivos de la psicología política (Hewer \& Lyons, 2018; Krosnick, Visser, \& Harder, 2010). La presente investigación se enmarca en esta línea y busca entender las actitudes de apoyo o rechazo que tuvo la ciudadanía chilena hacia el MECH durante el 2011 utilizando la Teoría de los Fundamentos Morales (TFM) (Graham et al., 2013; Graham et al., 2011). Esta teoría ha probado ser de utilidad para entender las actitudes hacia diversos temas sociopolíticos (Smith, Alford, Hibbing, Martin, \& Hatemi, 2017) como el aborto, el matrimonio homosexual, la pobreza o la desigualdad (Federico, Weber, Ergun, \& Hunt, 2013; Koleva, Graham, Iyer, Ditto, \& Haidt, 2012; Low \& Wui, 2016). Sin embargo, la mayoría de estos estudios se han dado en países culturalmente occidentales. En Latinoamérica, son muy pocos los estudios que han utilizado este marco para la investigación psicológica (Gudiño \& Fernandez, 2014; Silvino et al., 2016); por ello, la presente investigación no solo busca entender las actitudes sociopolíticas hacia el Movimiento, sino también brindar una oportunidad para observar la validez de la TFM en el contexto chileno.

\section{La teoría de los fundamentos morales}

La TFM señala que las personas y las culturas construyen su moralidad a partir de cinco sistemas psicológicos innatos, que estarían enraizados en nuestra evolución como especie (Haidt \& Graham, 2007; Haidt \& Joseph, 2004). Estos fundamentos son el daño/cuidado, la justicia/engaño, la lealtad/traición, la autoridad/ subversión y la santidad/degradación (Graham et al., 2013). El primer fundamento, de daño/ cuidado, está relacionado a nuestra historia evolutiva como mamíferos que ha modelado al cerebro humano para ser sensible a las señales de sufrimiento de otros (Haidt \& Graham, 2007). Este fundamento hace que las personas desaprueben a aquellos individuos que causan dolor o sufrimiento a otros, y aprueben a quienes previenen o alivian el dolor (Koleva et al., 2012). El fundamento de justicia/ engaño está relacionado a la evolución de los mecanismos de cooperación y el desarrollo de emociones que propician el altruismo recíproco, como el enojo, la culpa o la gratitud (Trivers, 1971). Este fundamento, también está asociado al valor de la justicia $y$, en las culturas occidentales, a valores como la equidad y los derechos individuales (Haidt \& Graham, 2007). Este fundamento debería hacer sensibles a las personas ante situaciones de injusticia y rechazar a aquellos que violan este principio (Koleva et al., 2012). El tercer fundamento, lealtad/traición estaría relacionado a nuestra historia como individuos tribales, los cuales compiten inter-grupalmente para sobrevivir; este fundamento valora los sacrificios que los individuos realizan por el grupo y se deplorará su traición (Haidt \& Graham, 2007); por lo que otros valores como la lealtad o el patriotismo están relacionados a este. Este fundamento también llevaría a las personas a aprobar las acciones de aquellos que contribuyen con la cohesión social y al bienestar del grupo (Koleva et al., 2012). El fundamento de autoridad/ 
subversión se relaciona a la tendencia, tanto de primates como humanos, de vivir y crear grupos sociales jerárquicamente estructurados. Valores como la obediencia, el respeto a la autoridad y el mantenimiento del orden son parte de este fundamento (Haidt \& Graham, 2007). Por último, el fundamento de santidad/degradación estaría basado en la emoción del asco (Rozin, Haidt, \& McCauley, 2008); la cual protegería al cuerpo de contaminantes biológicos ligados a la trasmisión de enfermedades. Asimismo, como emoción social, el asco se relaciona al rechazo a ciertas formas de apariencia u ocupaciones que también se pueden relacionar a enfermedades (Haidt \& Graham, 2007). En algunas culturas, el asco se ha relacionado a virtudes relacionadas a las actividades corporales y religiosas. Aquellos que se dejan llevar por las pasiones carnales serían vistos como impuros, mientras que aquellos que se dedican al cultivo del alma o la espiritualidad se les cataloga como elevados o santificados (Haidt, 2006).

Graham et al. (2013; 2011) Señalan que los fundamentos de daño/cuidado y de justicia/ engaño son fundamentos centrados en preservar la individualidad de la persona; mientras que los otros tres fundamentos son de cohesión social, ya que permiten a las personas permanecer juntas en grupos e instituciones grandes. Diferentes estudios han señalado la validez empírica de la TFM, utilizado para ello el Análisis Factorial Confirmatorio (CFA) para la propuesta de los cinco fundamentos morales, así como para la agrupación de los fundamentos individualizantes y de cohesión grupal (Davies, Sibley, \& Liu, 2014; Graham et al., 2011; Iurino \& Saucier, 2018; Nilsson \& Erlandsson, 2015; Silvino et al., 2016; Vainio \& Mäkiniemi, 2016; Yilmaz, Harma, Bahçekapili, \& Cesur, 2016). Sin embargo, los mismos autores reconocen que los índices estadísticos de bondad de ajuste no han sido robustos (Graham et al., 2011; Iurino \& Saucier, 2018).

\section{Fundamentos morales y actitudes políticas}

La TFM ha permitido entender muchos aspectos de la cognición y el comportamiento político (Graham, Haidt, \& Nosek, 2009; Haidt \& Graham, 2007; Smith et al., 2017). Por ejemplo, ha permitido comprender las diferencias psicológicas entre aquellos que se adscriben como liberales o conservadores en Estados Unidos (Silver \& Silver, 2017). De acuerdo a estos estudios, los liberales construyen su moral basados en los fundamentos de daño/ cuidado y de justicia/engaño; mientras que los conservadores valorarían de igual manera todos los fundamentos morales (Graham et al., 2009; Haidt \& Graham, 2007; Silver \& Silver, 2017). Es decir, la diferencia se encontraría en la valoración de los fundamentos de cohesión; los cuales son tomados en cuenta como elementos morales por los conservadores y no entendidos como tales por los liberales.

Partiendo de estos estudios, Koleva et al. (2012) señalan que los fundamentos morales serían las predisposiciones psicológicas subyacentes a las actitudes hacia temas sociopolíticos. Basados en esto, estos mismos autores encontraron que los fundamentos morales predicen las actitudes políticas de manera más potente que la orientación política y otras variables sociodemográficas (Koleva et al., 2012). De hecho, el fundamento de santidad/ degradación predijo las actitudes en nueve de los trece temas sociopolíticos examinados relacionados a la sexualidad, las relaciones de pareja y el matrimonio, y la santidad en la vida. En los últimos años, se ha incrementado el número de estudios que ha tomado la TFM para entender las actitudes sociopolíticas. Por ejemplo, Federico et al., (2013) encuentran que las actitudes hacia la igualdad se relacionan a los fundamentos individualizantes; mientras que las actitudes hacia la apertura o a la conformidad social estaban relacionadas a los fundamentos de cohesión social. Por otro lado, Low y Wui (2016) encuentran que los fundamentos morales son buenos predictores de las actitudes hacia 
la pobreza; donde, el daño/cuidado sería el más fuerte predictor.

\section{El Movimiento Estudiantil Chileno}

Si bien durante el año 2011 el MECh alcanza notoriedad internacional debido a las multitudinarias y enérgicas manifestaciones realizadas; este movimiento no aparece en un momento único, sino que es la historia de una generación que actúa en dos olas: la primera denominada "Revolución Pingüina" de 2006, en referencia al color blanco/negro de los uniformes escolares secundarios; y la segunda, llamada "Invierno Chileno" (Chilean Winter), haciendo alusión a la primavera árabe, esta vez protagonizada por estudiantes universitarios (Pedreira, 2014). Para comprender qué se entiende por MECh, su apoyo o rechazo, y cuáles son sus implicaciones para el contexto c, se describe brevemente el contexto social e histórico donde emerge.

La Ley Orgánica Constitucional de Enseñanza

1 fue la última ley promulgada por el régimen de Augusto Pinochet en Chile, y consolida legalmente la estructura del sistema educativo chileno. En consistencia con otras políticas de corte neoliberal implementadas durante este periodo de gobierno, el sistema educativo es mixto con una fuerte participación del sector privado y descentralización administrativa de la oferta estatal (Bellei \& Cabalin, 2013). Estas políticas específicas se implementan desde la predilección por el derecho de las familias a elegir una escuela, por sobre el derecho de las personas a una educación equitativa. El objetivo es que (1) la privatización de la educación y (2) la competencia entre escuelas, estimulada por mediciones estandarizadas frecuentes, decanten en un sistema de calidad que recoja de forma eficiente y se adapte a las necesidades de las familias chilenas (Bellei \& Cabalin, 2013; Pedreira, 2014). Es decir, detrás de estas políticas se asume que el mercado genere una educación de calidad en todos los niveles, incluyendo la educación superior (Bellei, Cabalin, \& Orellana, 2014).
Sin embargo, este sistema también ha tenido dos efectos que generaron malestar en la población, y motivaron las protestas del MECh: como generador de segregación y de endeudamiento (Bellei \& Cabalin, 2013; Pedreira, 2014). En ese sentido, el MECh toma como objetivos políticos la democratización del sistema de Educación Superior, a través de su regularización, que permita un acceso con equidad, calidad y heterogeneidad social a la matrícula universitaria (Urra, 2012).

\section{El presente estudio}

El MECh planteaba un cambio en la gestión del sistema educativo chileno; donde el Estado debe asegurar la igualdad de oportunidades para que los jóvenes chilenos puedan acceder al sistema educativo sin detrimento de la economía familiar y/o personal. En ese sentido, el MECh promueve una doctrina orientada a estimular la equidad y prevenir el daño a personas que no perciben ingresos económicos sustantivos (Urra, 2012). Específicamente, esperaríamos encontrar una relación positiva entre los fundamentos de justicia/engaño y daño/cuidado con el apoyo al MECh, y una relación negativa con los demás fundamentos. Además, se analizará si la orientación política puede mediar las relaciones entre los fundamentos morales y las actitudes sociopolíticas.

\section{Método}

\section{Participantes}

Se analizó la información de 170 personas ( 56.5 $\%$ de género masculino), todas de nacionalidad y residencia chilena, las cuales accedieron al cuestionario virtual a través de la plataforma de formularios de Google. El promedio de edad de la muestra fue de 27.85 años ( $S D=10.02$ años), en un rango de 15 años como edad mínima y 66 años como máxima. El $27.1 \%$ de la muestra señaló que residía en Santiago de Chile, región metropolitana, mientras que el resto residía en el 
interior del país. Un $44.1 \%$ de la muestra señaló contar con educación universitaria incompleta, un $27.6 \%$ con estudios universitarios completos, un $11.8 \%$ con estudios de pos-grado, un $8.2 \%$ a estudios técnicos completos o incompletos, y un $8.2 \%$ a estudios de educación media completos o incompletos. La mayoría de la muestra señaló que se adscribía al nivel socio-económico medio (47.6 $\%)$, seguido del nivel medio-bajo (22.9\%), y del medio-alto (18.8 \%). Un $32.5 \%$ señaló ser ateo o agnóstico, un 29 \% reportó profesar la religión católica, un $22.5 \%$ protestante o evangelista, y un $16 \%$ señaló profesar otras religiones.

\section{Instrumentos}

Orientación Política. Se indagó por la orientación política de los participantes a través de una escala de 5 puntos, donde 1 = "izquierda", 2 = "centroizquierda", 3 = "centro", 4 = "centro-derecha" y 5 = "derecha".

Nivel de Apoyo o Rechazo al Movimiento Estudiantil Chileno. Se indagó en los participantes por el nivel de apoyo o rechazo que tenían con respecto al MECh a través de una escala de 7 puntos, donde 1 = "Rechazo fuertemente al Movimiento Estudiantil" y $7=$ "Apoyo fuertemente al Movimiento Estudiantil".

Cuestionario de Fundamentos Morales. Instrumento de 30 ítems - MFQ30 (Graham et al., 2011), MoralFoundation.org - en su versión revisada y traducida al español. El cuestionario mide los fundamentos morales usando una escala de tipo Likert, y se encuentra divido en dos secciones. La primera, mide la relevancia que le da cada persona a ciertas consideraciones para decidir qué es lo bueno o lo malo; por ejemplo, "Si alguien sufre o no emocionalmente", la cual es medida en una escala que va del $0=$ "nada relevante" hasta el $6=$ "Muy relevante". La segunda sección mide el grado de acuerdo o desacuerdo con una serie de declaraciones morales; por ejemplo, "La compasión por los que sufren es la virtud más importante", la cual es medida en una escala que va del $0=$ "Muy en desacuerdo" hasta el 6 = "Muy de acuerdo". Las propiedades psicométricas fueron estudiadas por
Graham et al. (2011), los cuáles concluyen que se verifican los 5 fundamentos propuestos, con 6 ítems para cada uno de estos.

Para analizar la validez estructural del MFQ30, se realizó un Análisis Factorial Exploratorio (AFE) con todos los ítems, siguiendo las recomendaciones de Graham et al. (2011). De acuerdo a los valores autogenerados de Kaiser, se presentó una solución de 8 factores; sin embargo, el gráfico de sedimentación señaló una solución de dos factores. Al analizar la matriz de estructura, se observó que no todos los ítems se ajustaban a los factores esperados, la disposición de los mismos dificultaba su interpretación y ambos factores no llegaban a explicar el $30 \%$ de la varianza. Ante este escenario, se decidió realizar un AFE utilizando el método de Principales Componentes con una rotación Promax (Kim \& Mueller, 1978). Los valores autogenerados de Kaiser reportaban una solución similar de 8 factores, y de igual modo al observar el gráfico de sedimentación se concluyó en una solución de dos factores. En esta solución, todos los ítems cargaron a uno de los dos factores explicando el $33 \%$ de la varianza. Los fundamentos de autoridad/subversión y santidad/ degradación se agruparon en el primer factor; mientras que los fundamentos daño/cuidado y justicia/engaño se tendieron a agrupar en el segundo factor. El fundamento de lealtad/traición se dividió entre los dos factores con tres ítems para cada uno como se aprecia en la Tabla 1. 
Tabla 1

Análisis factorial exploratorio del MFQ - 30

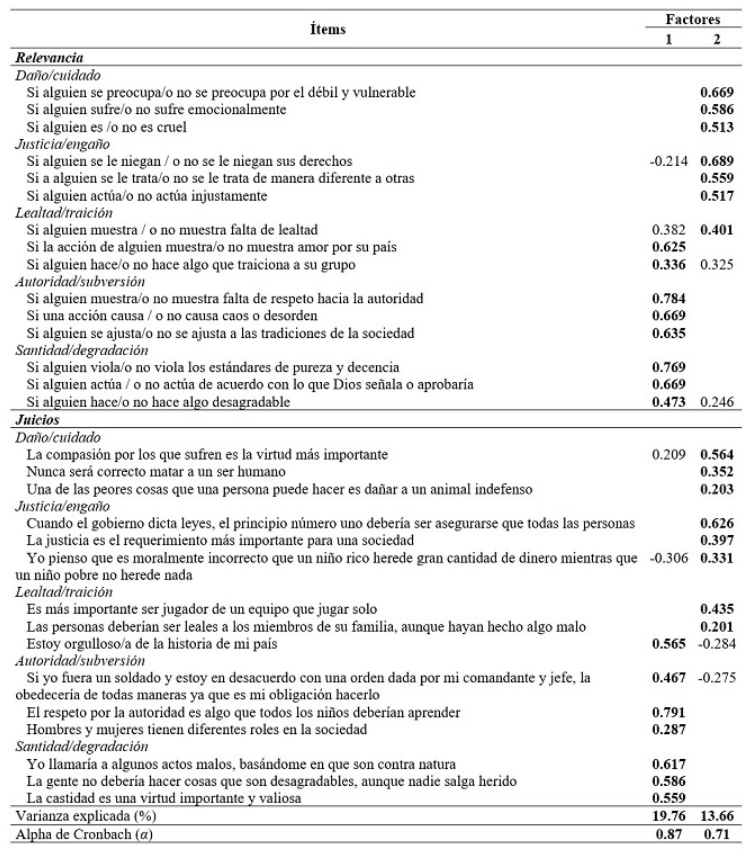

Los ítems de este fundamento referidos al amor por el país y al sentimiento de orgullo por la historia, asociables al valor del patriotismo, cargaron al primer factor sobre fundamentos de cohesión social. Los otros tres ítems relacionados a la lealtad, atribuyeron al segundo factor relacionado a los fundamentos individualizantes. Este resultado no es acorde a la TFM, pero consideramos que podría ser coherente con las características socio-culturales de la muestra estudiada. Es probable que los ítems relacionados al valor del patriotismo sean importantes para la muestra y su contexto; de hecho, este valor se ha relacionado en muestras chilenas a posiciones de autoritarismo de derecha (RWA) (Cárdenas \& Parra, 2010).

Luego, se procedió a realizar el Análisis Factorial Confirmatorio (AFC) del cuestionario, donde se contemplaba la posibilidad de un modelo de 5 factores similar al teórico planteado por Graham et al. (2011) y otro modelo de 6 factores, donde el fundamento de lealtad/ subvención se dividía en dos de acuerdo a los resultados del AFE: el de patriotismo (ítems referidos al orgullo por la historia y al amor al país) y el de lealtad (ítems referidos a la lealtad y la traición al grupo). Como se puede apreciar en la Tabla 2, el modelo de seis factores tuvo mejores índices de ajuste que el modelo de cinco factores, a pesar de que los índices del modelo de cinco factores coinciden con los propuestos por Graham et al (2011). Sin embargo, los índices en ambas estructuras fueron altos (Bollen, 1989; Browne \& Cudeck, 1992). Para mejorar este último modelo, se decidió eliminar los ítems que tenían coeficientes de regresión menores a 0.2 y no eran estadísticamente significativos; teniendo como resultado dos ítems eliminados, uno del factor de lealtad y otro del factor patriotismo.

\section{Figura 1}

Análisis Factorial Confirmatorio del MFQ30 de seis factores con items depurados

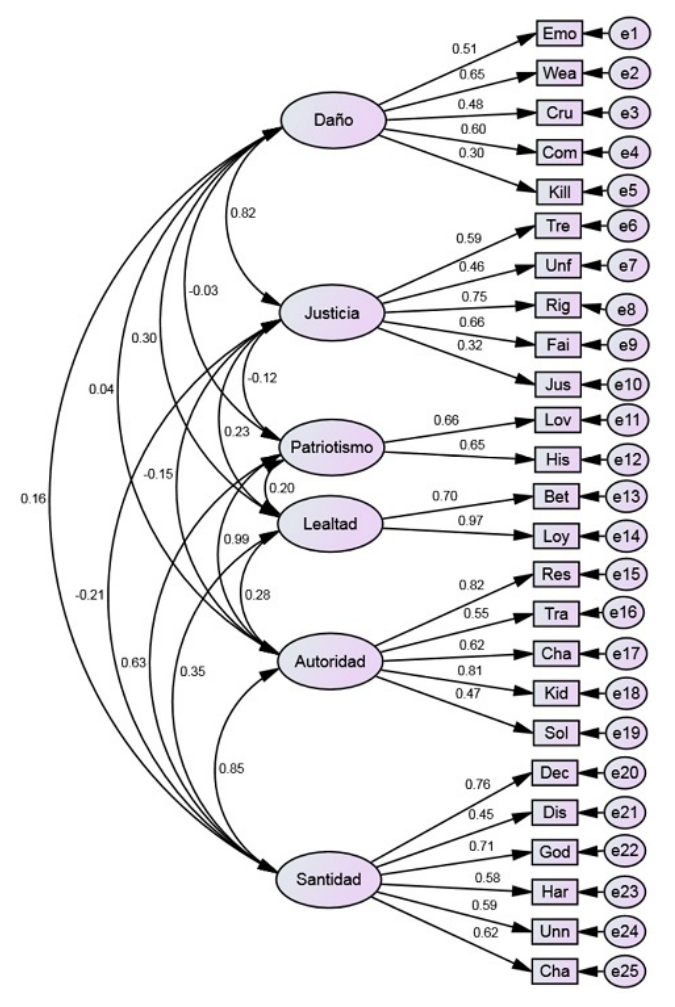

Los resultados, plasmados en la Tabla 2, muestran que el modelo de seis factores con ítems depurados tuvo mejores índices de ajuste que el modelo de cinco factores; además, éstos índices eran adecuados (Bollen, 1989; Browne $\&$ Cudeck, 1992 $)((260)=530.84)$, con un RMSEA $=0.079$, y un CFI $=0.81$ aceptable. Este modelo de seis factores con ítems depurados se utilizará para los análisis referidos al apoyo al 
MECh; modelo que tiene satisfactorios niveles de consistencia interna: $\alpha_{\text {daño/cuidado }}=0.59, \alpha_{\text {justicia/ }}$ engaño $=0.68, \quad \alpha_{\text {lealtad }}=0.81, \quad \alpha_{\text {patriotismo }}=0.60$, $\alpha_{\text {autoridad } / \text { subversión }}=0.79, \alpha_{\text {santidad } / \text { degradación }}=0.76$.

\section{Tabla 2}

Índices de bondad de ajuste de los modelos de cinco y seis factores del MFQ30

\begin{tabular}{ccccccccc}
\hline Estructura & $\boldsymbol{x}^{\mathbf{2}}$ & $\mathbf{d f}$ & $\boldsymbol{x}^{\mathbf{2}} / \boldsymbol{d} \boldsymbol{f} / \boldsymbol{d} \boldsymbol{f}$ & $\mathbf{N F I}$ & CFI & RMSEA & $\mathbf{9 0 \%} \mathbf{C I}$ \\
\hline Items Totales & & & & & & & \\
\hline Cinco factores & 942.67 & 395 & 2.39 & 0.538 & 0.659 & 0.091 & {$[0.083,0.098]$} \\
Seis factores & 904.64 & 390 & 2.32 & 0.557 & 0.680 & 0.088 & {$[0.081,0.096]$} \\
\hline Items depurados & & & & & & & \\
\hline Cinco factores & 648.74 & 265 & 2.45 & 0.626 & 0.732 & 0.093 & {$[0.084,0.102]$} \\
Seis factores & $\mathbf{5 3 0 . 8 4}$ & $\mathbf{2 6 0}$ & $\mathbf{2 . 0 4}$ & $\mathbf{0 . 6 9 4}$ & $\mathbf{0 . 8 1 1}$ & $\mathbf{0 . 0 7 9}$ & {$[\mathbf{0 . 0 6 9 , 0 . 0 8 8}]$} \\
\hline
\end{tabular}

\section{Procedimiento y Técnica Analítica}

Durante los meses de octubre y noviembre de 2011, en los cuales se desarrollaron las principales movilizaciones y protestas del MECh en Santiago de Chile, se habilitó a través de la plataforma de formularios Google, una encuesta de participación voluntaria que contenía el Cuestionario de Fundaciones Morales, así como otras preguntas relacionadas a las preferencias políticas, actitudes hacia el MECh y otras demográficas. Al inicio del cuestionario, cada participante era informado sobre los fines de la encuesta y que su participación era totalmente voluntaria; luego de ello, debía aceptar participar en esta y con ello iniciaba el diligenciamiento de la misma. A partir de los datos recolectados, se hizo una depuración de aquellos casos que, de acuerdo a lo recomendado por Graham et al. (2011), no debían ser tomados en cuenta. Luego de este proceso, se realizaron los análisis estadísticos para validar la estructura del Cuestionario de Fundamentos Morales. Primero, se realizaron análisis factoriales exploratorias y confirmatorias como se describe en la sección de instrumentos. Posteriormente, con los resultados de estos análisis se realizaron los procedimientos estadísticos inferenciales. En un primer momento, a través de análisis de correlaciones de Pearson y parciales. Para confirmar las relaciones encontradas en estos análisis, se desarrolló un modelo de ecuaciones estructurales (Skrondal \& Rabe-Hesketh, 2004), un método matemáticamente más riguroso que permite establecer relaciones entre las diferentes variables en un estudio.

\section{Resultados}

El análisis de correlaciones (Tabla 3) señala la fuerte relación entre el nivel de apoyo al MECh y la orientación política, señalando que aquellos que se posicionaban hacia la izquierda política tendían a dar mayor apoyo al MECh. Los fundamentos de patriotismo, autoridad/ subversión y santidad/degradación tuvieron una fuerte correlación negativa hacia el MECh y de manera positiva con la orientación política; sugiriendo que aquellos que puntuaron más alto en éstos tendían a rechazar al MECh e identificarse con la derecha política. De manera contraria, aquellos que puntuaron más alto en los fundamentos de daño/cuidado y justicia/engaño tendían a dar más apoyo al MECh y a posicionarse hacia la izquierda. Se realizó un análisis de correlaciones parciales para controlar el probable efecto de la orientación política, identificándose que compartía varianza con los fundamentos de justicia/engaño, autoridad/subversión y santidad/ degradación.

\section{Tabla 3}

Correlaciones bivariadas y parciales (1.b) entre fundamentos morales, orientación política, apoyo al MECh, y otras sociodemográficas

\begin{tabular}{|c|c|c|c|c|c|c|c|c|}
\hline Variables & 1 & 2 & 3 & 4 & 5 & 6 & 7 & 8 \\
\hline 1. Nivel de apoyo MECh & 1 & $-0.603^{* *}$ & $0.233^{* *}$ & $0.269^{* *}$ & -0.049 & $-0.4^{* *}$ & $-0.506^{* *}$ & $-0.382^{2 *}$ \\
\hline 2. Orientación Política & & 1 & -0.096 & $-0.241^{* *}$ & 0.122 & $0.405^{* *}$ & $0.573^{\mathrm{*m}}$ & $0.430^{* *}$ \\
\hline 3. Daño/cuidado & & & 1 & $0.559^{* *}$ & $0.201^{* s}$ & 0.008 & 0.027 & 0.132 \\
\hline 4. Justicia'engaño & & & & 1 & $0.161^{\circ}$ & -0.015 & -0.059 & -0.118 \\
\hline 5. Lealtad & & & & & 1 & $0.168^{*}$ & $0.252^{* *}$ & $0.294^{* *}$ \\
\hline 6. Patriotismo & & & & & & 1 & $0.688^{* \circ}$ & $0.419^{* *}$ \\
\hline 7. Autoridad/subversión & & & & & & & 1 & $0.660^{\circ *}$ \\
\hline 8. Santidad/degradación & & & & & & & & 1 \\
\hline Otras variables & & & & & & & & \\
\hline 9. Genero & 0.022 & -0.047 & 0.064 & 0.33 & 0.093 & 0.029 & 0.018 & -0.015 \\
\hline 10. Nivel Socioeconómico & $-0.203^{*}$ & $0.181 *$ & 0.076 & 0.055 & -0.04 & 0.029 & 0.057 & -0.025 \\
\hline $\begin{array}{l}\text { 1.b Nivel de apoyo } \\
\text { controlando Orientación } \\
\text { Política }\end{array}$ & & & $0.221^{* *}$ & $0.16^{*}$ & -0.031 & $-0.213^{* * *}$ & $-0.245^{* *}$ & $-0.170^{*}$ \\
\hline
\end{tabular}

Para determinar los factores subyacentes al apoyo o rechazo al $\mathrm{MECh}$, se desarrolló un modelo de ecuaciones estructurales, que permitió observar que los fundamentos de daño/ cuidado, patriotismo y santidad/degradación tenían una relación directa con el apoyo o rechazo al MECh; mientras que los fundamentos de justicia/engaño y autoridad/subversión tenían 
una relación mediada por la orientación política. El fundamento de daño/cuidado tuvo una influencia directa positiva, lo que señalaba que una mayor importancia de este fundamento tenía un efecto directo en el apoyo hacia el MECh. El fundamento de patriotismo y santidad/ degradación tenían una relación directa negativa con respecto al MECh; lo que señalaba que estos factores se relacionaban con el rechazo al movimiento. La relación entre el fundamento de justicia/engaño y el apoyo al MECh estuvo mediada por la orientación política; señalando que una mayor puntuación en el fundamento se relacionaba a posiciones políticas de izquierda, lo que a su vez influía en un mayor nivel de apoyo al MECh. Por último, el fundamento de autoridad/subversión también tenía una relación mediada por la orientación política; señalando que una mayor puntuación en este fundamento se asociaba a posiciones de derecha política, lo que a su vez se relacionaba con el rechazo al MECh.

\section{Figura 2}

Modelo de Ecuaciones Estructurales Fundamentos

Morales, Orientación Política y apoyo al MECh

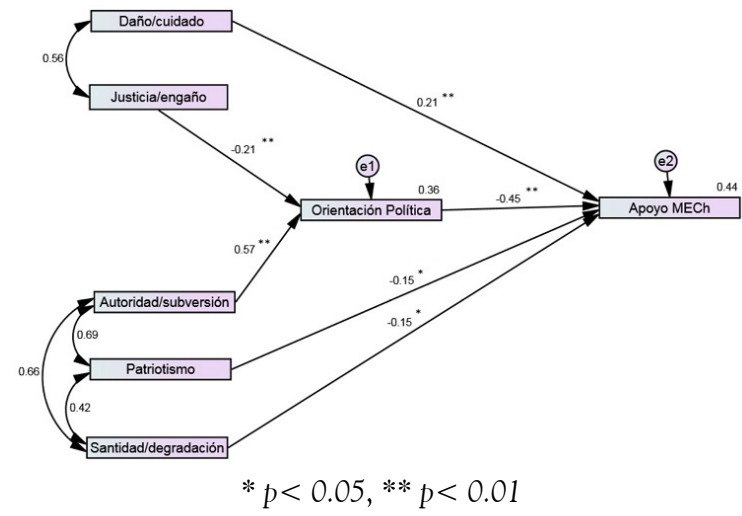

\section{Discusión}

La TFM propone una estructura de cinco fundamentos morales, innatos y universales, a partir de los cuales cada cultural desarrolla su sistema moral (Graham et al., 2013). La validación empírica por los autores de la TFM (Graham et al., 2011) y otros investigadores (Davies et al., 2014; Silvino et al., 2016; Vainio
\& Mäkiniemi, 2016; Yilmaz et al., 2016) se ha desarrollado principalmente a través de modelos de análisis factorial confirmatorios, los cuales no han tenido indicadores robustos. Este estudio es el primero en aplicar la TFM en una muestra chilena, lo que implica las limitaciones para generar el marco de este contexto. El estudio identificó que el modelo con mayor bondad de ajuste era de seis factores, el cual dividía el fundamento de lealtad/traición en dos valores, el de patriotismo y el de lealtad. Consideramos que estos resultados no invalidan la propuesta teórica, ya que la divergencia se da en un fundamento específico. Esta división resulta interesante ya que el valor del patriotismo ha sido relacionado a posiciones de derecha-autoritaria en muestras similares (Cárdenas \& Parra, 2010). Como se ve en los resultados del presente estudio existe una asociación similar, donde aquellos que se identifican de derecha política tienden a valorar más este factor que aquellos que se adscriben a la izquierda. El valor de lealtad no tuvo ningún efecto en términos de orientación política y del apoyo al MECh. Este resultado, podría interpretarse como un rasgo cultural asociado al colectivismo de este país (Hofstede \& Hofstede, 2005).

Por otro lado, este estudio confirma la utilidad de la TFM para el análisis de la cognición política, señalando que los fundamentos morales subyacerían a diferentes actitudes socio-políticas (Koleva et al., 2012). Los fundamentos referidos a la justicia/engaño y daño/cuidado se relacionaban con posiciones políticas de izquierda y a un mayor apoyo al MECh; mientras que la autoridad/subversión, la santidad/degradación y el patriotismo, se relacionaban con posiciones de derecha y a un menor apoyo al MECh. Mediante los análisis de ecuaciones estructurales se pudo determinar que la orientación política mediaba las relaciones entre los fundamentos de justicia/engaño y autoridad/subversión con el apoyo o rechazo al MECh; mientras que el daño/cuidado, el patriotismo y la santidad/ degradación tenían una relación directa en el apoyo o rechazo al MECh. Con respecto al fundamento de justicia/engaño, el que no todas las personas tengan la oportunidad de 
acceder a una carrera universitaria o superior por temas socioeconómicos podría verse como una injusticia que hay que rechazar, ya que va en contra del valor de la igualdad, el cual se ha relacionado a posiciones de izquierda (Thorisdottir, Jost, Liviatan, \& Shrout, 2007) y lo que coincide con la evidencia recogida. Con respecto al fundamento de daño/cuidado, el cual tenía una relación directa hacia el apoyo al MECh; ésta tiene sentido en la medida que se perciba que el hecho de que una persona no pueda acceder a la educación superior debido al alto costo económico como una forma de daño, por lo que es importante apoyar el MECh para prevenirlo o aliviarlo. Con respecto al fundamento de autoridad/subversión, para aquellos que puntuaron alto en este, el MECh pudo verse como un elemento que ponía en riesgo las estructuras sociales y el orden establecido, por lo que les causaba rechazo. Existe amplia evidencia que ha asociado estos elementos con posiciones de derecha (Caprara et al., 2017; Haidt \& Graham, 2007; Piurko, Schwartz, \& Davidov, 2011; Schwartz, Caprara, \& Vecchione, 2010), por lo que queda justificado lo obtenido en el análisis de ecuaciones estructurales.

Por último, esta investigación nos habla de la importancia de analizar los conflictos sociales y políticos en la región desde el marco de la psicología moral; que permite comprender las concepciones morales de los individuos, elemento clave del procesamiento de la información social, que dirige las actitudes de los individuos hacia diferentes temas sociopolíticos, incluyendo sus posturas hacia políticas públicas y movimientos sociales. Una de las consecuencias de la divergencia de actitudes enraizadas en valores o en visiones distintas de lo moral, es que los conflictos sociales que se originan son muy álgidos. Como plantean los autores de la TFM para explicar la "guerra cultural americana" (Graham, 2009); en el presente estudio la TFM sirve para observar cuáles son los valores morales subyacentes a las opiniones hacia el MECh y entender por qué existían posturas irreconciliables entre quienes lo apoyaban y entre quienes lo rechazaban; ya que sus posiciones se basaban en entendimientos de la moral diferentes. Asimismo, esto también explica la larga duración que tuvo este conflicto social en Chile; ya que las protestas no solo se extendieron durante el año 2011, sino que inclusive estas demandas de un sistema educativo basado en la igualdad continuaron durante el gobierno sucesor de Michelle Bachellet (Larrabure \& Torchia, 2015). Por ello, se hace cada vez más necesaria la incorporación de las ciencias cognitivas en el desarrollo de políticas públicas, la comunicación política, la gestión de los conflictos y el entendimiento de la opinión pública. Estos elementos, pueden ayudar a entender mejor el impacto de las políticas públicas en el bienestar de las personas, y, con ello, su pertinencia y sostenibilidad. Como algunos autores lo han planteado, las políticas neoliberales implementadas por Pinochet en materia de educación generaron un malestar en la sociedad, debido a las inequidades sociales que se producían por este sistema; malestar que se canalizó a través del MECh (Mayol Miranda \& Azócar Rosenkranz, 2011; Pousadela, 2013). En ese sentido, la TFM nos ayuda a entender que el apoyo al MECh estaba basado en principios relacionados a la justicia, en términos de equidad, y al cuidado; a diferencia de posturas más conservadoras, en las cuales prevalecía una posición de preservar el statu quo (Cabalin, 2014).

Estudios a futuro deben profundizar el rol de los fundamentos morales en la gestación de los conflictos políticos, la elaboración de políticas públicas y el surgimiento de los movimientos sociales. Existe evidencia que los fundamentos morales permiten explicar diversas actitudes sociopolíticas (Smith et al., 2017). Esto es fundamental en el contexto Latinoamericano, en donde las posiciones políticas de los gobiernos de la región han cambiado de estar marcadas hacia la izquierda a haber girado hacia la derecha en los últimos años (Zovatto et al., 2018). Además de que para construir democracias más sólidas, es fundamental entender a la ciudadanía. Asimismo, estos estudios futuros deben superar las limitaciones del presente, especialmente en cuanto a alcanzar muestras representativas en las poblaciones a estudiar. 


\section{Referencias}

Bellei, C., \& Cabalin, C. (2013). Chilean student movements: Sustained struggle to transform a market-oriented educational system. Current Issues in Comparative Education, 15(2), 108-123. Recuperado de https://www.tc.columbia.edu/cice/pdf/2 8175 15 02 Bellei_Cabalin.pdf

Bellei, C., Cabalin, C., \& Orellana, V. (2014). The 2011 Chilean student movement against neoliberal educational policies. Studies in Higher Education, 39(3), 426-440. https://doi.org/10.1080/03075079 .2014 .896179

Bollen, K. A. (1989). A new incremental fit index for general structural equation models. Sociological Methods Eु Research, 17(3), 303-316. https://doi.org/10.1177/00491241 89017003004

Browne, M. W., \& Cudeck, R. (1992). Alternative ways of assessing model fit. Sociological Methods $\mathcal{E}$ Research, 21(2), 230-258. https://doi.org/10.1177/00491241 92021002005

Cabalin, C. (2014). The conservative response to the 2011 Chilean student movement: Neoliberal education and media. Discourse: Studies in the Cultural Politics of Education, 35(4), 485-498. https://doi.org/10.1080/01 596306.2013 .871233

Caprara, G. V., Vecchione, M., Schwartz, S. H., Schoen, H., Bain, P. G., Silvester, J., . . . Caprara, M. G. (2017). Basic values, ideological self-placement, and voting: A cross-cultural study. CrossCultural Research, 51 (4), 388-411. https://d oi.org/10.1177/1069397117712194

Cárdenas, M., \& Parra, L. (2010). Adaptación y validación de la Versión Abreviada de la Escala de Autoritarismos de Derechas (RWA) en una muestra chilena. Revista de Psicología, 19(1), 61-79. https://doi.org/10.5 354/0719-0581.2011.17098

Centro de Estudios de la Realidad Contemporánea. (2011). Barómetro de la Política - Agosto Setiembre. Recuperado de Santiago de
Chile: http://www.cooperativa.cl/noticias/s ite/artic/20110927/asocfile/201109271201 24/1364157_1.pdf

Davies, C. L., Sibley, C. G., \& Liu, J. H. (2014). Confirmatory factor analysis of the moral foundations questionnaire. Social Psychology, 45(6), 431-436. https://doi.org /10.1027/1864-9335/a000201

Federico, C. M., Weber, C. R., Ergun, D., \& Hunt, C. (2013). Mapping the connections between politics and morality: The multiple sociopolitical orientations involved in moral intuition. Political Psychology, 34(4), 589-610. https://doi.org/10.1111/pops.120 06

Graham, J., Haidt, J., Koleva, S., Motyl, M., Iyer, R., Wojcik, S. P., \& Ditto, P. H. (2013). Chapter two - Moral foundations theory: The pragmatic validity of moral pluralism. In P. Devine \& A. Plant (Eds.), Advances in Experimental Social Psychology (Vol. 47, pp. 55-130). https://doi.org/10.1016/B9780-12-407236-7.00002-4

Graham, J., Haidt, J., \& Nosek, B. A. (2009). Liberals and conservatives rely on different sets of moral foundations. Journal of personality and social psychology, 96(5), 1029-1046. https://doi.org/10.1037/a00151 41

Graham, J., Nosek, B. A., Haidt, J., Iyer, R., Koleva, S., \& Ditto, P. H. (2011). Mapping the moral domain. Journal of personality and social psychology, 101 (2), 366-385. https://d oi.org/10.1037/a0021847

Gudiño, S., \& Fernandez, J. (2014). Los fundamentos morales en bachillerato, un estudio exploratorio. Revista de Investigacion Educativa, 5(9), 44-49. Recuperado de https://www.rieege.mx/index.php/rieege /article/viewFile/180/83

Haidt, J. (2006). The happiness hypothesis: Finding modern truth in ancient wisdom. New York: Basic Books.

Haidt, J., \& Graham, J. (2007). When morality opposes justice: Conservatives have moral intuitions that liberals may not recognize. Social Justice Research, 20(1), 98-116. https ://doi.org/10.1007/s11211-007-0034-z 
Haidt, J., \& Joseph, C. (2004). Intuitive ethics: How innately prepared intuitions generate culturally variable virtues. Daedalus, 133(4), 55-66. https://doi.org/10.1162/001 1526042365555

Hewer, C. J., \& Lyons, E. (2018). Political psychology : A social psychological approach. Newark: John Wiley \& Sons, Incorporated. https://doi.org/10.1002/9781118982365

Hofstede, G., \& Hofstede, G. J. (2005). Cultures and organizations. New York: McGraw-Hill.

Iurino, K., \& Saucier, G. (2018). Testing Measurement Invariance of the Moral Foundations Questionnaire Across 27 Countries. Assessment. https://doi.org/10.1 177/1073191118817916

Kim, J.-O., \& Mueller, C. W. (1978). Factor analysis: Statistical methods and practical issues (Vol. 14). Thousand Oaks, CA: Sage Publications. https://doi.org/10.4135/9781 412984256

Koleva, S. P., Graham, J., Iyer, R., Ditto, P. H., $\&$ Haidt, J. (2012). Tracing the threads: How five moral concerns (especially Purity) help explain culture war attitudes. Journal of research in personality, 46(2), 184-194. ht tps://doi.org/10.1016/j.jrp.2012.01.006

Krosnick, J. A., Visser, P. S., \& Harder, J. (2010). The psychological underpinnings of political behavior. In S. Fiske, D. Gilbert \& G. Lendzey (Eds.), Handbook of Social Psychology (Vol. II). New Jersey: John Wiley \& Sons, Inc. https://doi.org/10.1002/97804 70561119.socpsy002034

Larrabure, M., \& Torchia, C. (2015). The 2011 Chilean student movement and the struggle for a new left. Latin American Perspectives, 42 (5), 248-268. https://doi.org $/ 10.1177 / 0094582 X 14547506$

Low, M., \& Wui, M. G. L. (2016). Moral foundations and attitudes towards the poor. Current Psychology, 35(4), 650-656. https:// doi.org/10.1007/s12144-015-9333-y

Mayol Miranda, A., \& Azócar Rosenkranz, C. (2011). Politización del malestar, movilización social y transformación ideológica: el caso "Chile 2011". Polis,
10(30), 163-184. https://doi.org/10.4067/S 0718-65682011000300008

Nilsson, A., \& Erlandsson, A. (2015). The moral foundations taxonomy: Structural validity and relation to political ideology in Sweden. Personality and Individual Differences, 76, 28-32.https://doi.org/10.10 16/j.paid.2014.11.049

Pedreira, M. (2014). El movimiento estudiantil chileno: Análisis de las demandas de los estudiantes y del impacto político de las movilizaciones. (Unpublished undergraduate thesis). Universitat Autònoma de Barcelona, Barcelona. Recuperado de https://dd.uab. cat/record/119181

Piurko, Y., Schwartz, S. H., \& Davidov, E. (2011). Basic personal values and the meaning of left-right political rientations in 20 Countries. Political Psychology, 32(4), 537-561. https://doi.org/10.1111/j.1467-92 21.2011.00828.x

Pousadela, I. M. (2013). Protest and proposal, participation and representation: the Chilean student movement, 2011-12. Development in Practice, 23(5-6), 685-700. https://doi.org/10.1080/09614524 .2013 .800837

Rozin, P., Haidt, J., \& McCauley, C. R. (2008). Disgust. In M. Lewis, J. M. Haviland-Jones \& L. F. Barrett (Eds.), Handbook of emotions, (3rd ed., pp. 757-776). New York, NY: Guilford Press. Recuperado de https://cpb-u s-w2.wpmucdn.com/web.sas.upenn.edu/dis t/7/206/files/2016/09/DgLewisHbk2008-psi by3.pdf

Schwartz, S. H., Caprara, G. V., \& Vecchione, M. (2010). Basic personal values, core political values, and voting: A longitudinal analysis. Political Psychology, 31 (3), 421-452. https:// doi.org/10.1111/j.1467-9221.2010.00764.x

Silver, J. R., \& Silver, E. (2017). Why Are Conservatives More Punitive Than Liberals? A Moral Foundations Approach. Law and Human Behavior, 41 (3), 258-272. http://dx.doi.org/10.1037/hb0000232

Silvino, A. M. D., Pilati, R., Keller, V. N., Silva, E. P., Freitas, A. F. d. P., Silva, J. N., \& Lima, 
M. F. (2016). Adaptação do questionário dos fundamentos morais para o português. Psico-USF, 21(3), 487-495. http://dx.doi.or g/10.1590/1413-82712016210304

Skrondal, A., \& Rabe-Hesketh, S. (2004). Generalized latent variable modeling: Multilevel, longitudinal, and structural equation models. Boca Raton, Fla., London: CRC. https://doi.org/10.1201/9780203489 437

Smith, K. B., Alford, J. R., Hibbing, J. R., Martin, N. G., \& Hatemi, P. K. (2017). Intuitive ethics and political orientations: Testing moral foundations as a theory of political ideology. American Journal of Political Science, 61 (2), 424-437. https://doi .org/10.1111/ajps.12255

Thorisdottir, H., Jost, J. T., Liviatan, I., \& Shrout, P. E. (2007). Psychological needs and values underlying left-right political orientation: Cross-national evidence from Eastern and Western Europe. Public Opinion Quarterly, 71(2), 175-203. https://doi.org/10.1093/po $\mathrm{q} / \mathrm{nfm} 008$

Trivers, R. L. (1971). The evolution of reciprocal altruism. The Quarterly Review of Biology, 46(1), 35-57. https://doi.org/doi:10.1086/4 06755

Urra, J. (2012). La movilización estudiantil chilena en 2011: Una cronología. Revista del Observatorio Social de América Latina, 13(31), 23-38.

Vainio, A., \& Mäkiniemi, J.-P. (2016). How are moral foundations associated with climate-friendly consumption? Journal of Agricultural and Environmental Ethics, 29(2), 265-283. https://doi.org/10.1007/s10806-0 $16-9601-3$

Yilmaz, O., Harma, M., Bahçekapili, H. G., \& Cesur, S. (2016). Validation of the moral foundations questionnaire in Turkey and its relation to cultural schemas of individualism and collectivism. Personality and Individual Differences, 99, 149-154. http s://doi.org/10.1016/j.paid.2016.04.090

Zovatto, D., Arana Araya, I., Rojas Silva, N., Mijares, V. M., García Pinzón, V., \& Duarte-Recalde, L. R. (2018). El gran ciclo electoral latinoamericano. Iberoamericana, 18(69), 227-254. http://dx.doi.org/10.1844 1/ibam.18.2018.69.227-254

\section{Notas}

\footnotetext{
* Artículo de investigación.

1 Ley n. ${ }^{\circ}$ 18.962, Orgánica Constitucional de Enseñanza (LOCE), elaborada por la Junta de Gobierno y promulgada por el dictador Augusto Pinochet el 10 de marzo de 1990.
} 